\title{
Improved Health-related Quality of Life and Physical Function in Patients with Refractory Chronic Gout Following Treatment with Pegloticase: Evidence from Phase III Randomized Controlled Trials
}

\author{
VIBEKE STRAND, DINESH KHANNA, JASVINDER A. SINGH, ANNA FORSYTHE, and N. LAWRENCE EDWARDS
}

\begin{abstract}
Objective. To assess the efficacy of pegloticase on pain, physical function, and health-related quality of life (HRQOL) in patients with refractory chronic gout.

Methods. Subjects in 2 replicate, 6-month, randomized controlled phase III trials received intravenous infusions of pegloticase $8 \mathrm{mg}$ twice monthly (biweekly group), pegloticase alternating with placebo (8-mg monthly group), or placebo. Medical Outcomes Study Short Form-36 (SF-36), Health Assessment Questionnaire-Disability Index (HAQ-DI), patient global assessment of disease activity (PtGA), and pain by visual analog scale were completed at weeks 1 (baseline), 13, 19, and 25. Prespecified pooled analyses of patient-reported outcomes were performed by combining values for each treatment group (biweekly treatment, monthly treatment, and placebo) at Week 25.

Results. Of 212 patients enrolled, 157 (74.1\%) completed treatment. At entry, mean age was 55.4 years (range 23-89 yrs) and mean plasma uric acid was $9.7 \mathrm{mg} / \mathrm{dl}$; most were male $(81.6 \%$ ) and white (67.5\%). Subjects reported an average of 9.8 flares in the previous 18 months. Baseline SF-36 physical component summary (PCS) scores were $>1.5$ SD below US normative values. At Week 25 , mean changes from baseline in PtGA, pain, HAQ-DI, and PCS scores were statistically significant and exceeded minimum clinically important differences (MCID) in the biweekly treatment group, compared with little to no improvement in placebo group. Statistically significant improvements greater than or equal to MCID were reported in 6 of 8 SF-36 domains. Monthly pegloticase resulted in significantly improved PtGA, HAQ-DI, PCS, and 3 SF-36 domains.

Conclusion. Pegloticase therapy resulted in statistically significant and clinically meaningful improvements in PtGA, pain, physical function, and HRQOL. (First Release June 1 2012; J Rheumatol 2012;39:1450-7; doi:10.3899/jrheum.111375)
\end{abstract}

Key Indexing Terms:

PEGLOTICASE GOUT OUTCOMES RESEARCH TREATMENT PATIENT PERSPECTIVE

Refractory chronic gout (RCG) occurs in patients who have failed to normalize levels of serum uric acid (SUA), and in

From the Division of Immunology and Rheumatology, Stanford University School of Medicine, Portola Valley, California; Division of Rheumatology, Department of Internal Medicine, University of Michigan Health System, Ann Arbor, Michigan; Medicine Service, Center for Chronic Disease Outcomes Research, University of Alabama, Birmingham, Alabama; Health Economics and Outcomes Research, Savient Pharmaceuticals Inc., East Brunswick, New Jersey; and Department of Rheumatology and Clinical Immunology, University of Florida, Gainesville, Florida, USA. Supported by Savient Pharmaceuticals Inc., East Brunswick, NJ, USA. V. Strand, MD, Division of Immunology and Rheumatology, Stanford University School of Medicine; D. Khanna, MD, MS, Division of Rheumatology, Department of Internal Medicine, University of Michigan Health System; J.A. Singh, MD, MPH, Medicine Service, Center for Chronic Disease Outcomes Research, University of Alabama; A. Forsythe, PharmD, Health Economics and Outcomes Research, Savient Pharmaceuticals Inc.; N.L. Edwards, MD, Department of Rheumatology and Clinical Immunology, University of Florida.

Address correspondence to Dr. V. Strand, Division of Immunology and Rheumatology, Stanford University School of Medicine, 306 Ramona Road, Portola Valley, CA 94028, USA. E-mail: vstrand@stanford.edu Full Release Article. For details see Reprints/Permissions at jrheum.org Accepted for publication March 21, 2012. whom signs and symptoms are inadequately controlled with conventional urate-lowering therapies (ULT) at maximum medically appropriate doses or for whom ULT are contraindicated. RCG is a severe form of arthritis with signs and symptoms that may include frequent and prolonged flares, presence of tophi, chronically tender and swollen joints, and impaired physical function and health-related quality of life $(\mathrm{HRQOL})^{1}$. Refractory disease is estimated to occur in roughly 120,000 of the 3 million to 6 million Americans who experience gout based on a recent comprehensive market research study (unpublished data, Savient Pharmaceuticals Inc.).

The clinical course and therapeutic management of patients with RCG can be further complicated by the presence of significant medical comorbidities, most commonly hypertension, metabolic syndrome, chronic kidney disease, and osteoarthritis (OA), and less frequently cardiovascular disorders and diabetes ${ }^{1}$. Epidemiologic studies show that these patients are at increased risk of both cardiovascular events and all-cause mortality ${ }^{2}$. When RCG occurs in the presence of 
multiple comorbidities, the associated polypharmacy can make patients exceptionally difficult to manage.

RCG has a major influence on HRQOL, affecting physical as well as emotional and social functioning, particularly in patients with medical comorbidities ${ }^{1,3,4}$. Both Medical Outcomes Study Short Form-36 (SF-36) and Health Assessment Questionnaire-Disability Index (HAQ-DI) have been validated in patients with chronic gout ${ }^{1,5}$. Studies in patients with gout and RCG have reported considerably impaired HRQOL compared with published norms for age and $\operatorname{sex}^{1,3}$. In comparison with published results in other chronic conditions, SF-36 scores in subjects with RCG were equivalent to those in patients with longstanding rheumatoid arthritis (RA) or active lupus erythematosus, and worse than in US subjects with hypertension and $\mathrm{OA}^{4}$. In a 52-week longitudinal observational study of 110 patients with RCG, HRQOL was substantially worse in those with severe rather than mild to moderate disease, and the number of flares and tender and swollen joints correlated with impaired HRQOL and physical function $^{1}$. Presence of tophi also correlated with worse HRQOL. Importantly, HRQOL scores in these patients with RCG did not improve over the 12-month observational period despite maximal medical management by rheumatologists.

Pegloticase is a PEGylated recombinant mammalian uricase used for treatment of patients with $\mathrm{RCG}^{6}$. Pegloticase catalyzes oxidation of uric acid into 5-hydroxyisourate, which spontaneously converts to the soluble metabolite, allantoin, which is readily eliminated primarily through renal clearance. As a result, pegloticase administration reduces SUA or plasma uric acid (PUA) levels below limits of solubility (SUA or PUA $<6 \mathrm{mg} / \mathrm{dl}$ ). This is thought to create a urate concentration gradient that draws extravascular urate into the circulation for enzymatic degradation. Over time, reduction in extravascular urate favors dissolution of urate crystals and normalization of body urate pools, leading to improvements in signs and symptoms of gout and resolution of tophi ${ }^{7,8}$.

The efficacy and tolerability of biweekly or monthly pegloticase treatment in patients with RCG were demonstrated in 2 replicate, 6-month, randomized controlled phase III trials $(\mathrm{RCT})^{9}$. The primary endpoint - treatment response defined as SUA $<6.0 \mathrm{mg} / \mathrm{dl}$ for $80 \%$ of the time in months 3 and 6 - was achieved by significantly more patients who received treatment with biweekly or monthly pegloticase compared with placebo ( $42 \%$ and $35 \%$ vs $0 \%$, respectively; $\mathrm{p}<0.001$ for each comparison). Our report summarizes the effects of pegloticase treatment on patient-reported outcomes (PRO) from these 2 trials, including patient global assessment of disease activity (PtGA) and pain, physical function, and HRQOL, prespecified as a pooled analysis of both RCT by treatment group at Week 25.

\section{MATERIALS AND METHODS}

The design of the phase III RCT was as reported ${ }^{9}$. Briefly, adults with refractory gout characterized by baseline SUA $\geq 8.0 \mathrm{mg} / \mathrm{dl} ; \geq 3$ self-reported gout flares during the previous 18 months, $\geq 1$ tophi, and/or gouty arthropathy; and either contraindication to allopurinol treatment or failure to normalize uric acid after $\geq 3$ months of treatment with the maximum medically appropriate allopurinol dose were enrolled. Patients were randomly allocated to receive 12 biweekly 2-h intravenous infusions of pegloticase $8 \mathrm{mg}$ (q2 week or biweekly treatment group), pegloticase $8 \mathrm{mg}$ alternating with placebo at successive infusions (monthly treatment group), or placebo. Prior ULT were discontinued during a 1-week washout. Prophylaxis for gout flares (colchicine or a nonsteroidal antiinflammatory drug) was started 1 week before the first infusion and continued throughout the study, and prophylaxis against infusion-related reactions (fexofenadine, acetaminophen, and hydrocortisone) was administered before each infusion. A total of 225 subjects were randomized in the 2 RCT; 212 subjects received at least 1 infusion of study treatment ( 85 in the pegloticase biweekly treatment group, 84 in the pegloticase monthly treatment group, and 43 in the placebo group) and were included in the modified intent-to-treat population. Of these, 157 subjects completed protocol treatment.

Patient-reported outcomes. PRO were evaluated as secondary outcomes in the RCT. Patients completed the following at baseline and at weeks 13, 19 , and 25: PtGA and pain using a 100-mm visual analog scale (VAS), physical function by HAQ-DI, and HRQOL by SF-36. Results for HAQ-DI and SF-36 are presented for subjects with data available at baseline and weeks 13, 19, and 25. For each PRO, the minimum clinically important difference (MCID) represents the level of improvement that is perceptible to patients, defined as $\geq 10$ points on a 100 -mm VAS ${ }^{10,11}$. HAQ-DI has been used extensively as a measure of physical function in RCT and longitudinal observational studies and was demonstrated to fulfill the Outcome Measures in Rheumatology filter in gout ${ }^{4,12}$. An improvement of -0.22 in HAQ-DI score is considered to represent MCID in patients with RA and other rheumatologic conditions ${ }^{4,13,14}$. SF-36 is a validated measure of HRQOL or "multidimensional function," and has been used extensively in RCT and longitudinal observational studies evaluating a variety of medical conditions including $\mathrm{RA}^{13,15}$, $\mathrm{OA}^{16}$, systemic lupus erythematosus ${ }^{17,18}$, psoriatic arthritis ${ }^{19}$, systemic sclerosis $^{20}$, fibromyalgia ${ }^{21}$, and most recently gout ${ }^{1,3,9,22}$. It includes 8 domains: physical functioning (PF), role physical (RP), bodily pain (BP), general health $(\mathrm{GH})$, vitality (VT), social functioning (SF), role emotional (RE), and mental health $(\mathrm{MH})$, which are aggregated into physical component summary (PCS) and mental component summary (MCS) scores. The PCS score positively weights the 4 physical domains PF, RP, BP, and GH, as well as VT, negatively weighting the remaining mental domains. The MCS score positively weights the 4 mental domains VT, RE, SF, and $\mathrm{MH}$ and negatively weights the physical domains. Improvements of 5-10 points in domain scores and 2.5-5 points in PCS and MCS scores represent MCID in rheumatologic conditions based on correlations with PtGA and/or the Guyatt "feeling thermometer" $11,23,24,25,26$.

Health utilities by the Short Form-6D (SF-6D) were computed from SF-36 at baseline and endpoint based on mean scores across all 8 domains, as recently validated by Ara and Brazier ${ }^{27}$ and validated against EuroQoL 5 dimensions ${ }^{28}$. Utilities are scored from 0 (death) to 1 (perfect health), with the minimally important difference $(\mathrm{MID})=0.041^{27}$.

The proportion of subjects reporting improvements greater than or equal to MCID in each PRO was analyzed. To determine the relationship between the pharmacodynamic effects of pegloticase and treatment-associated benefits, longterm responders with sustained normalization of PUA receiving biweekly pegloticase were analyzed according to the number of outcomes with reported improvements greater than or equal to MCID.

Statistical analysis. Demographic and baseline characteristics were compared across treatment groups (biweekly vs monthly treatment and placebo) by ANOVA for continuous variables and chi-square test for categorical measures. Prespecified pooled analyses of the PRO from the replicate phase III RCT were performed by combining values for each treatment group (biweekly treatment, monthly treatment, and placebo) at Week 25. Age- and sexmatched norms were calculated for the combined groups. To facilitate comparisons, change scores were used to derive baseline scores for each treatment group, and then the mean of these values was calculated after correcting for the imbalance in randomization (i.e., 2:2:1) to derive a single baseline score 
for the entire population. As data were normally distributed, pairwise comparisons of PtGA, pain, HAQ-DI, and SF-36 PCS, MCS, and domain scores between each pegloticase treatment group versus placebo were performed using a 2-sample $t$ test. Mean changes from baseline were analyzed using a linear model with treatment as the fixed factor and baseline value as a covariate. Within-group comparisons at different timepoints were made using a 1 -sample $t$ test. Fisher's exact test compared treatment differences in responder rates for each and for combined PRO. SF-36 data are presented as spydergrams, which offer a simplified means to visualize changes across all domains in a single figure. The spydergrams depict disease- and populationspecific patterns of decrements in HRQOL compared with age- and sex-matched normative data, as well as providing a tool for interpreting complex treatment-associated or longitudinal changes ${ }^{15}$.

\section{RESULTS}

Subjects enrolled in the 2 RCT had advanced disease, associated, on average, with 10 acute gout flares during the 18-month period before study entry (Table 1). Seventy-three percent had tophi; $63 \%$ considered their gout flares to be severe/crippling; and 58\% had chronic pain and synovitis/arthropathy. This
RCG population was also characterized by a high prevalence of medical comorbidities: $84 \%$ had at least 1 coexisting cardiovascular condition/risk factor, $61 \%$ were obese, and $28 \%$ had chronic kidney disease (Table 1).

PtGA and pain VAS scores ranged from 42 to 54 across treatment groups at baseline, indicative of moderate to severe disease activity. Mean HAQ-DI scores in the pegloticase biweekly, pegloticase monthly, and placebo groups were 1.10, 1.21 , and 1.24 , respectively, comparable to those reported in patients with longstanding active RA ${ }^{4}$. Baseline SF-36 HRQOL scores were low across treatment groups; PCS scores were $>1.5 \mathrm{SD}$ below the US normative value of 50, with values of 35.2 in the pegloticase biweekly group, 33.3 in the pegloticase monthly group, and 31.0 in the placebo group (Table 2). Domain scores, with the exception of MH, were also 12 to 32 points lower than age- and sex-matched normative US population values (Table 2). The baseline SF-6D score in the pooled population was 0.656 compared with the

Table 1. Baseline characteristics of the pooled population from the 2 randomized controlled trials.

\begin{tabular}{|c|c|c|c|}
\hline Characteristic & $\begin{array}{l}\text { oticase Biweekly, } \\
\qquad \mathrm{n}=85\end{array}$ & $\begin{array}{l}\text { Pegloticase Monthly, } \\
\qquad \mathrm{n}=84\end{array}$ & $\begin{array}{l}\text { Placebo, } \\
\mathrm{n}=43\end{array}$ \\
\hline \multicolumn{4}{|l|}{ Demographics } \\
\hline Age, mean yrs & 56.3 & 54.5 & 55.4 \\
\hline Male sex, $\mathrm{n}(\%)$ & $68(80)$ & $69(82)$ & $36(84)$ \\
\hline White race/ethnicity, n (\%) & $54(64)$ & $59(70)$ & $30(70)$ \\
\hline Body mass index, mean $\mathrm{kg} / \mathrm{m}^{2}$ & 33 & 33 & 32 \\
\hline \multicolumn{4}{|l|}{ Allopurinol } \\
\hline Contraindication, n (\%) & $69(81)$ & $67(80)$ & $38(88)$ \\
\hline Ineffective, $\mathrm{n}(\%)$ & $16(19)$ & $17(20)$ & $5(12)$ \\
\hline \multicolumn{4}{|l|}{ Gout-related characteristics } \\
\hline Duration, mean yrs & 15 & 16 & 13 \\
\hline Acute flares in prior 12 mo, mean* & 6.5 & 6.4 & 6.8 \\
\hline Baseline tophi present, $\mathrm{n}(\%)$ & $62(73)$ & $64(76)$ & $29(67)$ \\
\hline Chronic synovitis or arthropathy, n (\%) & $50(59)$ & $47(56)$ & $26(61)$ \\
\hline HAQ-DI, mean & 1.10 & 1.21 & 1.24 \\
\hline Plasma uric acid, mean, mg/dl & 9.8 & 9.9 & 9.2 \\
\hline \multicolumn{4}{|l|}{ Baseline comorbid conditions, n (\%) } \\
\hline $\begin{array}{l}\text { One or more cardiovascular conditions or } \\
\text { risk factors } * *\end{array}$ & $72(85)$ & $71(85)$ & $35(81)$ \\
\hline Hypertension & $62(73)$ & $60(74)$ & $31(72)$ \\
\hline Dyslipidemia & $42(49)$ & $41(49)$ & $20(46)$ \\
\hline Diabetes mellitus & $24(38)$ & $18(21)$ & $8(19)$ \\
\hline Cardiac arrhythmia & $19(22)$ & $8(9)$ & $7(16)$ \\
\hline Coronary artery disease & $14(16)$ & $16(19)$ & $9(21)$ \\
\hline Cardiac failure/LVD & $12(14)$ & $8(9)$ & $6(14)$ \\
\hline Peripheral vascular disease & $7(8)$ & $6(7)$ & $3(7)$ \\
\hline Cerebrovascular disease & $4(5)$ & $3(4)$ & $1(2)$ \\
\hline Obesity, BMI $\geq 30 \mathrm{~kg} / \mathrm{m}^{2}$ & $50(59)$ & $55(65)$ & $24(59)$ \\
\hline Osteoarthritis & $29(34)$ & $23(27)$ & $16(37)$ \\
\hline Chronic kidney disease $* * *$ & $26(30)$ & $25(30)$ & $9(21)$ \\
\hline Sleep apnea & $8(9)$ & $9(11)$ & $6(14)$ \\
\hline Venous thromboembolic disease & $3(3)$ & $2(2)$ & $2(5)$ \\
\hline
\end{tabular}

* Self-reported. ** Includes any of the following: hypertension, dyslipidemia, diabetes, cardiac arrhythmias, coronary artery disease, cardiac failure, left ventricular dysfunction, peripheral vascular disease, or cerebrovascular disease. $* * *$ Chronic kidney disease was defined as creatinine clearance $<60 \mathrm{ml} / \mathrm{min}$ calculated with the Cockcroft-Gault formula ${ }^{29}$. BMI: body mass index; LVD: left ventricular dysfunction; HAQ-DI: Health Assessment Questionnaire Disability Index. 
Table 2. Comparison of baseline SF-36 domain scores in randomized controlled trials (RCT) with age/sex-matched US norms, as well as other refractory chronic gout populations.

\begin{tabular}{|c|c|c|c|c|c|c|c|c|}
\hline \multirow{2}{*}{ Study Group } & \multicolumn{8}{|c|}{ Domain } \\
\hline & $\mathrm{PF}$ & $\mathrm{RP}$ & $\mathrm{BP}$ & $\mathrm{GH}$ & VT & SF & $\mathrm{RE}$ & $\mathrm{MH}$ \\
\hline \multicolumn{9}{|l|}{ RCT trial population and matched US norms, score source } \\
\hline Age/sex-matched US norms* & 77.5 & 76.7 & 67.5 & 67.1 & 58.8 & 81.8 & 84.2 & 75.0 \\
\hline Decrements in baseline scores compared with age/sex norms & 32.3 & 29.5 & 30.1 & 19.7 & 11.5 & 19.9 & 14.8 & 4.4 \\
\hline \multicolumn{9}{|l|}{ Comparable refractory chronic gout populations, score source } \\
\hline $65-74$ yrs with OA/HTN ${ }^{\dagger \dagger}$ & 69.4 & 64.5 & 68.5 & 62.6 & 59.9 & 80.6 & 81.4 & 76.9 \\
\hline Angina + HTN $(59.7 \text { yrs })^{\dagger \dagger}$ & 63.3 & 44.2 & 61.6 & 52.0 & 48.5 & 80.3 & 70.2 & 73.0 \\
\hline
\end{tabular}

* Based on age and sex distribution of the RCT population ${ }^{30}$. ** Patients $(n=110)$ had treatment-failure gout, defined as symptomatic crystal-proven gout with duration $\geq 2$ years, and intolerance or refractoriness to conventional urate-lowering therapy as indicated by serum uric acid $>6.0 \mathrm{mg} / \mathrm{dl}$; mean age 59 years, male $81.8 \%$, white $68.2 \%{ }^{1}$. ${ }^{\dagger}$ Veterans in Veterans Administration database with ICD-9 diagnosis of gout who responded to survey and had 1 or more arthritic conditions; demographic characteristics of this subset not reported ${ }^{3}$. Total number of survey respondents with gout was 1090 . ${ }^{\dagger \dagger}$ From SF-36 manu$\mathrm{al}^{30}$. BP: bodily pain; GH: general health; MH: mental health; PF: physical functioning; RE: role emotional; RP: role physical; SF: social functioning; VT: vitality; NHS: National Health Service; VHA: Veterans Health Administration; OA: osteoarthritis; HTN: hypertension; SF-36: Medical Outcomes Study Short Form-36.

age- and sex-matched US normative value of 0.825 , reflecting the major effects of disease on HRQOL.

The pharmacodynamic effects of pegloticase treatment on PUA and tophi reported previously ${ }^{9}$ were associated with statistically significant and clinically meaningful improvements in PRO, including PtGA, pain, physical function, and HRQOL. In the prespecified pooled analysis, biweekly treatment with pegloticase was associated with statistically significant mean improvements from baseline in PtGA, pain, HAQ-DI, and PCS scores, each of which exceeded MCID, compared with little to no improvement with placebo treatment (Table 3, Figure 1). Monthly peglot- icase also significantly improved PtGA, HAQ-DI, and PCS scores.

In the pooled analysis of SF-36, significant improvements were reported in 6 of 8 domains with biweekly pegloticase and 3 of 8 domains with monthly pegloticase (Table 4, Figure 2). With biweekly pegloticase treatment, the largest changes were observed in BP, RP, SF, and PF. Domain scores at Week 25 met or exceeded US age- and sex-matched norms in VT and $\mathrm{MH}$, and approached normative values in $\mathrm{BP}, \mathrm{SF}$, and RE. In comparison, changes in SF-36 domain scores with placebo ranged from worsening ( -1.13 in $\mathrm{BP})$ to improvement (4.6 in $\mathrm{RE}$ ), none of which met MCID (Table 4, Figure 2). As base-

Table 3. Number (\%) of subjects with improvements from baseline greater or equal to minimum clinically important differences.

\begin{tabular}{lccc}
\hline Measure & $\begin{array}{c}\text { Pegloticase } \\
\text { Biweekly }\end{array}$ & $\begin{array}{c}\text { Pegloticase } \\
\text { Monthly }\end{array}$ & Placebo \\
\hline Patient global assessment & 50 & 57 & 35 \\
$\mathrm{~N}^{*}$ & $27(54)$ & $29(51)$ & $10(29)$ \\
$\mathrm{N}(\%)$ final visit & 0.03 & $\mathrm{NS}$ & \\
$\mathrm{p}$ vs placebo** & & & \\
Pain & 60 & 62 & 37 \\
$\mathrm{~N}$ & $33(55)$ & $\mathrm{NS}$ & \\
$\mathrm{N}(\%)$ final visit & 0.01 & 63 & 38 \\
$\mathrm{p}$ vs placebo** & 62 & $30(48)$ & $6(16)$ \\
Health Assessment Questionnaire-Disability Index & $<0.001$ & \\
$\mathrm{~N}$ & $28(45)$ & & 38 \\
$\mathrm{~N}(\%)$ final visit & $<0.003$ & 62 & $11(29)$ \\
$\mathrm{p}$ vs placebo** & 58 & $38(61)$ & \\
Physical Component Summary & 58 & $<0.01$ & \\
$\mathrm{~N}$ & $37(64)$ & $<.01$ & \\
$\mathrm{~N}(\%)$ Week 25 & $<01$ & \\
$\mathrm{p}$ vs placebo** & & & \\
\hline
\end{tabular}

* Number of subjects with both baseline and Week 25 data. ** Fisher's exact test to compare rate of responders in pegloticase treatment versus placebo. 


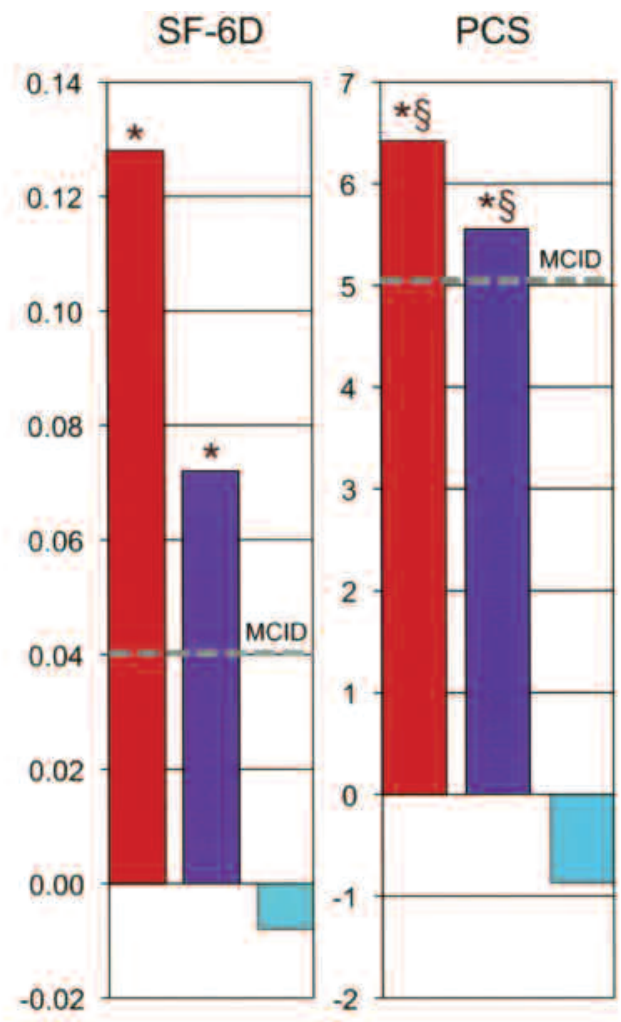

Pegloticase biweekly
Patient Global

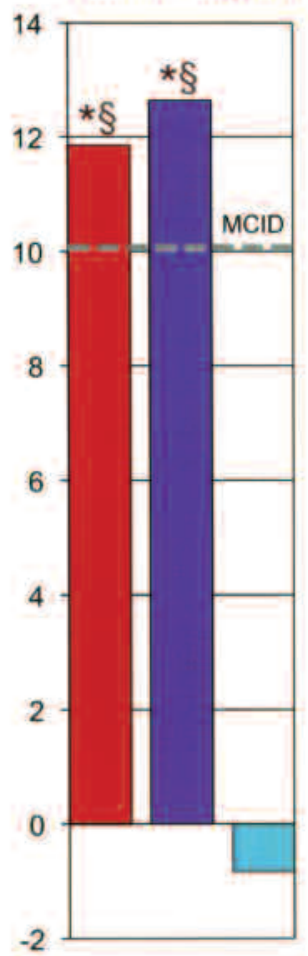

Pegloticase monthly
Pain

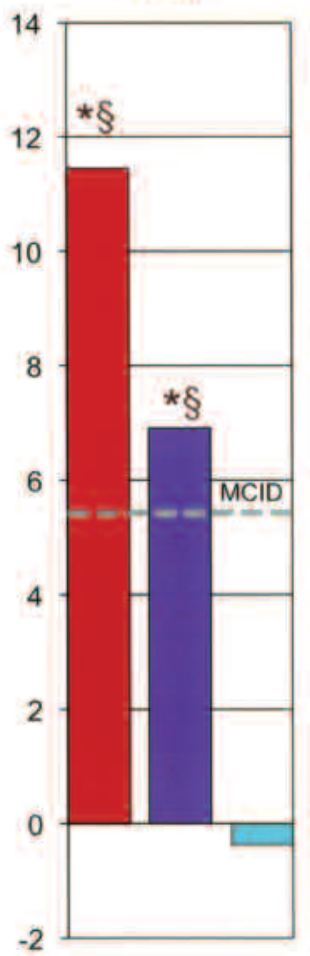

HAQ-DI

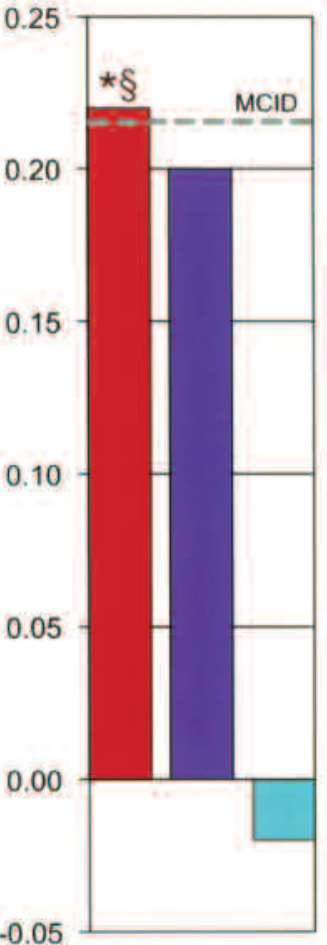

Placebo

*Improvements $\geq$ MCID (minimum clinically important difference) §P-value vs. placebo $<0.05$

Figure 1. Mean change in patient-reported outcomes from baseline to Week 25 with biweekly or monthly pegloticase compared with placebo. SF-6D: preference-based single index health measure; PCS: physical component summary; HAQ-DI: Health Assessment Questionnaire-Disability Index; MCID: minimum clinically important difference.

Pegloticase (8 mg monthly) vs Age/Gender Norms

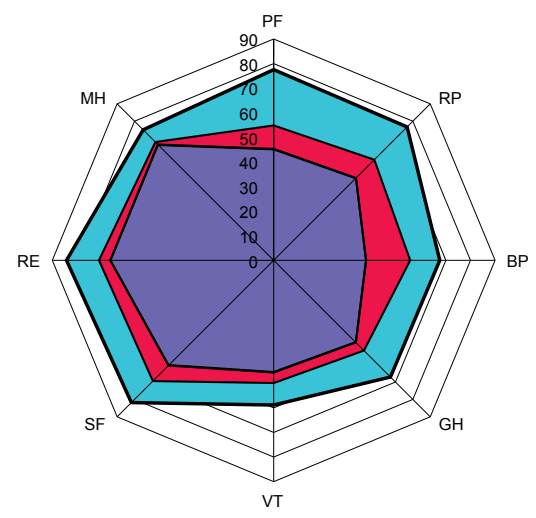

$\square$ Age/Gender Norms

$\square$ Pegloticase 8 mg monthly - Week 25

$\square$ Pegloticase $8 \mathrm{mg}$ monthly - Baseline(25)
Pegloticase (8 mg biweekly) vs Age/Gender Norms

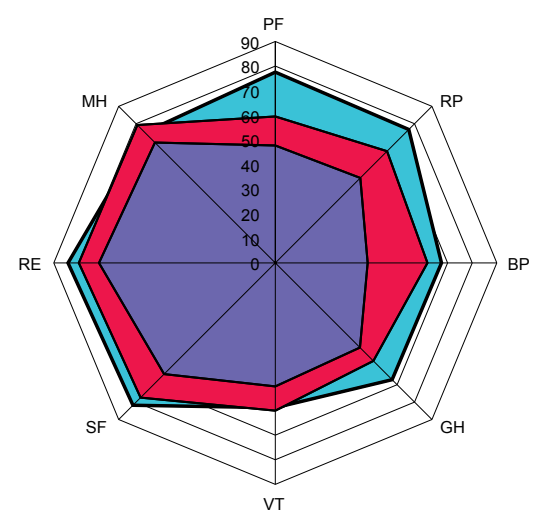

$\square$ Age/Gender Norms

$\square$ Pegloticase $8 \mathrm{mg}$ biweekly - Week 25

$\square$ Pegloticase $8 \mathrm{mg}$ biweekly - Baseline(25)
Placebo vs Age/Gender Norms

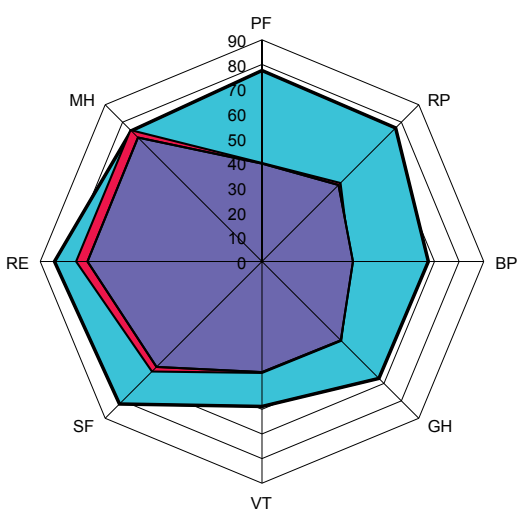

$\square$ Age/Gender Norms

$\square$ Placebo - Week 25

$\square$ Placebo - Baseline(25)

Figure 2. Clinically meaningful improvement in Medical Outcomes Study Short Form-36 domain scores from baseline to Week 25 with biweekly and monthly pegloticase. Domain scores are plotted from 0 (worst) at the center to 90 (best) at the outer edge. Physical function (PF) is plotted at the top followed clockwise by role physical (RP), bodily pain (BP), general health $(\mathrm{GH})$, vitality (VT), social functioning (SF), role emotional (RE), and mental health (MH). Gridlines along axes represent changes of 10 points, equivalent to 1-2 times minimal clinically important difference. The inner polygon (purple) represents baseline domain scores and the outer polygon (aqua) shows age- and sex-matched norms; the intermediate polygon (red) represents scores at Week 25 in the 2 pegloticase groups. Domain scores are connected by lines to facilitate recognition of patterns, and not to imply that these are continuous scales. Differences in the shape of the octagonal patterns provide a graphic representation for comparing baseline domain values with age/sex normative values and for evaluating treatment-associated changes from baseline to Week 25 . 
Table 4. Mean ( \pm SD) change in SF-36 domain scores from baseline to Week 25. Shaded area shows changes in domain scores that are greater than or equal to minimum clinically important differences.

\begin{tabular}{lcccccccc}
\hline Domain & PF & RP & BP & GH & VT & SF & RE & MH \\
\hline Pegloticase biweekly, & $11.8^{* \dagger}$ & $15.4^{* \dagger}$ & $24.3^{* \dagger}$ & $7.7^{*}$ & $9.9^{*}$ & $13.5^{* \dagger}$ & 8.2 & 10.1 \\
$\mathrm{n}=61^{\dagger \dagger}$ & $(24.1)$ & $(27.8)$ & $(25.5)$ & $(17.5)$ & $(20.1)$ & $(28.9)$ & $(30.6)$ & $(19.2)$ \\
Pegloticase monthly, & $9.5^{*}$ & $10.5^{*}$ & $17.9^{*}$ & 4.7 & 4.3 & 8.9 & 4.6 & 1.4 \\
$\mathrm{n}=63^{\dagger \dagger}$ & $(20.3)$ & $(28.6)$ & $(24.2)$ & $(17.2)$ & $(20.1)$ & $(26.5)$ & $(30.3)$ & $(16.8)$ \\
Placebo, $\mathrm{n}=38^{\dagger \dagger}$ & 0.25 & 1.15 & -1.13 & 0.26 & 0.33 & 2.63 & 4.61 & 4.3 \\
& $(18.97)$ & $(20.90)$ & $(20.77)$ & $(14.97)$ & $(15.24)$ & $(23.99)$ & $(22.40)$ & $(18.1)$ \\
\hline
\end{tabular}

\footnotetext{
* p values $<0.05$ based on independent-groups t tests of means for treatment groups compared to placebo.

$\dagger$ Approached or met age/sex-matched normative values. ${ }^{\dagger}$ Number of subjects at Week 25; only patients with complete data through Week 25 are included in this analysis. BP: bodily pain; GH: general health; MH: mental health; PF: physical functioning; RE: role emotional; RP: role physical; SF: social functioning; VT: vitality.
}

line MCS scores were similar to age- and sex-matched population norms, little improvement was expected or observed in any treatment group.

Improvements from baseline to Week 25 in SF-6D utility scores were $0.128(19.6 \%)$ with biweekly pegloticase and $0.072(11.0 \%)$ with monthly pegloticase, both of which exceeded MID. In comparison, the SF-6D utility score deteriorated in the placebo group by 0.008 .

\section{DISCUSSION}

The phase III RCT demonstrated that biweekly pegloticase the US Food and Drug Administration (FDA)-approved schedule for treatment of chronic gout refractory to conventional therapy ${ }^{31}$ - produced rapid normalization of PUA values and reductions in tophus burden, which were associated with fewer gout flares ${ }^{9}$. Our present report extends these findings, demonstrating that pegloticase administration also improves PRO. With biweekly pegloticase, patients reported statistically significant improvements in PtGA, pain, physical function, SF-36 PCS, and 6 of 8 SF-36 domain scores - not only physical domains but also VT (reflecting fatigue, energy, and pep) and social functioning. Of note, SF-36 scores at Week 25 in the biweekly pegloticase group approached or met age- and sex-matched normative values in 5 of 8 domains, with the largest improvements in those domains associated with the lowest scores at baseline. These results clearly reflect improvements in multidimensional functioning that are both statistically significant and clinically meaningful with biweekly pegloticase treatment.

Monthly pegloticase also improved PRO, although the effects were not as robust as in the biweekly treatment group. Statistically significant improvements with monthly pegloticase were seen in PtGA, HAQ-DI, PCS, and 3 domains of the SF-36. Although biweekly and monthly pegloticase had generally similar effects on the efficacy variables in these phase III trials, the subjects receiving monthly pegloticase had more infusion reactions and early treatment discontinuations, which may have contributed to the differences in PRO between treatment groups ${ }^{9}$.

Chronic gout, in general, is associated with marked detri- mental effects on HRQOL and physical function. Patients with RCG are among those with the greatest burden of disease, and consequently have significant need for effective treatment. Improvements demonstrated with pegloticase, while more pronounced on the physical domains, were still evident on the mental domains of the SF-36, suggesting that RCG affects physical function, pain, and fatigue, as well as how a subject feels emotionally and interacts socially. These data indicate that pegloticase treatment resulted in important and clinically meaningful improvements not only in physical functioning and bodily pain but also in fatigue, emotional status, and social interactions.

The presence of comorbidities such as hypertension, chronic kidney disease, OA, and other medical conditions complicates the management of RCG by limiting use of some therapies. In addition, polypharmacy in these patients can further limit attempts to control gout. Given that impairment of HRQOL in patients with RCG is also related to the presence of comorbidities ${ }^{32}$, the importance of significant improvements in HRQOL demonstrated by pegloticase treatment in these phase III RCT should not be underestimated.

Although treatments for chronic gout have been available for many years, none to date has been shown to be effective in improving HRQOL and physical function ${ }^{1}$. Accordingly, the improvement in these PRO with pegloticase is noteworthy. Moreover, the improvements in HRQOL and physical function reported with biweekly pegloticase treatment over 6 months are comparable in magnitude to those reported with infliximab plus methotrexate (MTX) and adalimumab plus MTX in patients with longstanding RA who had failed multiple disease-modifying antirheumatic drugs ${ }^{15}$.

Improvements in disease symptoms as well as HRQOL and physical function reported here may be expected to lead to increases in work productivity and societal benefits. Bruce and Fries reported significant associations between HAQ-DI and productivity, morbidity, healthcare use, healthcare costs, and death ${ }^{33}$. Similarly, Fleishman, et al determined that SF-36 PCS values had substantial predictive ability for medical expenditure ${ }^{34}$. Based on the findings in other conditions, the magnitude of changes in physical function, HRQOL, and Personal non-commercial use only. The Journal of Rheumatology Copyright @ 2012 . All rights reserved. 
health utilities in patients with RCG receiving pegloticase therapy could be expected to translate into meaningful savings in direct medical costs and cumulative treatment costs.

Cost-effectiveness is an important factor when choosing between various interventions. Health utility measures can be used to determine cost-effectiveness, and the SF-6D health utility allows a quantitative measure of improvement across all 8 domains of SF-36. Baseline SF-6D score in the pooled treatment population was 0.656 , similar to that of other RCG cohorts (e.g., 0.679 in the Natural History Study and 0.629 in the Veterans Health Administration Survey ${ }^{1,3,32}$ ). Improvements in SF-6D utilities in the pegloticase treatment groups (0.128 with biweekly pegloticase and 0.072 with monthly pegloticase) were consistent with improvements in other PRO (e.g., PtGA, pain, and HAQ-DI), exceeded the MCID, and were comparable to or better than improvements in patients with established RA treated for 6 months with adalimumab and MTX combination therapy $(0.06)^{27}$.

The number needed to treat (NNT) for a given therapy is the reciprocal of the absolute risk reduction or improvement generated by that treatment. The NNT is helpful to clinicians and payers, enabling them to translate results from RCT and systematic reviews into clinical practice. The NNT with biweekly pegloticase was 1.2 (95\% CI 1.1 to 1.4$)$, indicating that nearly every patient who receives and can tolerate biweekly pegloticase for 6 months will achieve a clinically meaningful improvement in at least 1 PRO.

Treatment with the FDA-approved schedule of biweekly pegloticase results in statistically significant and clinically meaningful improvements in pain, global assessment of disease activity, physical function, and HRQOL in patients with RCG.

\section{ACKNOWLEDGMENT}

The authors thank Richard Brook, MS, MBA, The JeSTARx Group, Newfoundland, NJ, USA, for his comments on the drafts of this report.

\section{REFERENCES}

1. Becker MA, Schumacher HR, Benjamin KL, Gorevic P, Greenwald $\mathrm{M}$, Fessel J, et al. Quality of life and disability in patients with treatment-failure gout. J Rheumatol 2009;36:1041-8.

2. Roddy E, Doherty M. Epidemiology of gout. Arthritis Res Ther 2010;12:223.

3. Singh JA, Strand V. Gout is associated with more comorbidities, poorer health-related quality of life and higher healthcare utilisation in US veterans. Ann Rheum Dis 2008;67:1310-6.

4. Strand V, Singh JA. Improved health-related quality of life with effective disease-modifying antirheumatic drugs: Evidence from randomized controlled trials. Am J Manag Care 2008;14:234-54.

5. Alvarez-Hernández E, Peláez-Ballestas I, Vázquez-Mellado J, Terán-Estrada L, Bernard-Medina AG, Espinoza J, et al. Validation of the Health Assessment Questionnaire disability index in patients with gout. Arthritis Rheum 2008;59:665-9.

6. Sherman MR, Saifer MG, Perez-Ruiz F. PEG-uricase in the management of treatment-resistant gout and hyperuricemia. Adv Drug Deliv Rev 2008;60:59-68.

7. Pascual E, Sivera F. Time required for disappearance of urate crystals from synovial fluid after successful hypouricaemic treatment relates to the duration of gout. Ann Rheum Dis 2007;66:1056-8.

8. Perez-Ruiz F, Calabozo M, Pijoan JI, Herrero-Beittes AM, Ruibal A. Effect of urate-lowering therapy on the velocity of size reduction of tophi in chronic gout. Arthritis Rheum 2002;15:356-60.

9. Sundy JS, Baraf HSB, Yood RA, Edwards NL, Gutierrez-Urena SR, Treadwell EL, et al. Efficacy and tolerability of pegloticase for the treatment of chronic gout in patients refractory to conventional treatment. Two randomized controlled trials. JAMA 2011;306:711-20.

10. Wells GA, Tugwell P, Kraag GR, Baker PR, Groh J, Redelmeier DA. Minimum important difference between patients with rheumatoid arthritis: The patient's perspective. J Rheumatol 1993;20:557-60.

11. Beaton DE, Boers M, Wells GA. Many faces of the minimal clinically important difference (MCID): A literature review and directions for future research. Curr Opin Rheumatol 2002;14:109-14.

12. Bruce B, Fries JF. The Stanford Health Assessment Questionnaire: A review of its history, issues, progress, and documentation. J Rheumatol 2003;30:167-78.

13. Tugwell P, Wells G, Strand V, Maetzel A, Bombardier C, Crawford $\mathrm{B}$, et al. Clinical improvement as reflected in measures of function and health-related quality of life following treatment with leflunomide compared with methotrexate in patients with rheumatoid arthritis: Sensitivity and relative efficiency to detect a treatment effect in a twelve-month, placebo-controlled trial. Leflunomide Rheumatoid Arthritis Investigators Group. Arthritis Rheum 2000;43:506-14. Erratum in: Arthritis Rheum 2000;43:1345.

14. Wolfe F, Michaud K, Strand V. Expanding the definition of clinical differences: from minimally clinically important differences to really important differences. Analyses in 8931 patients with rheumatoid arthritis. J Rheumatol 2005;32:583-9.

15. Strand V, Singh JA. Newer biological agents in rheumatoid arthritis: Impact on health-related quality of life and productivity. Drugs 2010;70:121-45.

16. Strand V, Kelman A. Outcome measures in osteoarthritis: Randomized controlled trials. Curr Rheumatol Rep 2004;6:20-30.

17. Strand V, Petri M, Buyon J, Joh T, Freimuth W, Sigler L, et al. Systemic lupus erythematosus impacts all domains of health-related quality of life (HRQOL): Baseline results from five randomized controlled trials [abstract]. Ann Rheum Dis 2007;66:SII:482.

18. Thumboo J, Strand V. Health-related quality of life in patients with systemic lupus erythematosus: An update. Ann Acad Med Singapore 2007;36:115-22.

19. Strand V, Sharp V, Koenig A, Park G, Shi Y, Wang B, et al. Comparison of health-related quality of life in rheumatoid arthritis, psoriatic arthritis and psoriasis and effects of etanercept treatment. Ann Rheum Dis 2012 Jan 17 [E-pub ahead of print].

20. Khanna D, Furst DE, Clements PJ, Park GS, Hays RD, Yoon J, et al. Responsiveness of the SF-36 and the Health Assessment Questionnaire Disability Index in a systemic sclerosis clinical trial. J Rheumatol 2005;32:832-40.

21. Choy EH, Arnold LM, Clauw DJ, Crofford LJ, Glass JM, Simon LS, et al. Content and criterion validity of the preliminary core dataset for clinical trials in fibromyalgia syndrome. J Rheumatol 2009;36:2330-4.

22. Khanna D, Ahmed M, Yontz D, Ginsburg SS, Park GS, Leonard A, et al. The disutility of chronic gout. Qual Life Res 2008;17:815-22.

23. Angst F, Aeschlimann A, Stucki G. Smallest detectable and minimal clinically important differences of rehabilitation intervention with their implications for required sample sizes using 
WOMAC and SF-36 quality of life measurement instruments in patients with osteoarthritis of the lower extremities. Arthritis Rheum 2001;45:384-91.

24. Strand V. Longer term benefits of treating rheumatoid arthritis: Assessment of radiographic damage and physical function in clinical trials. Clin Exp Rheumatol 2004;22:S57-64

25. Kosinski M, Zhao SZ, Dedhiya S, Osterhaus JT, Ware JE Jr. Determining minimally important changes in generic and disease-specific health-related quality of life questionnaires in clinical trials of rheumatoid arthritis. Arthritis Rheum 2000;43:1478-87.

26. Strand V, Crawford B. Improvement in health-related quality of life in patients with systemic lupus erythematosus following sustained reductions in anti-dsDNA antibodies. Exp Rev Pharmacoecon Outcomes Res 2005;5:317-26.

27. Ara R, Brazier J. Predicting the Short Form-6D Preference-Based Index using the eight mean Short Form-36 Health Dimension scores: Estimating preference-based health-related utilities when patient level data are not available. Value Health 2009;12:346-53.

28. Ara R, Brazier J. Deriving an algorithm to convert the eight mean SF-36 dimension scores into a mean EQ-5D preference-based score from published studies (where patient level data are not available). Value Health 2008;11:1131-43.
29. Cockcroft DW, Gault MH. Prediction of creatinine clearance from serum creatinine. Nephron 1976;16:31-41.

30. Ware JE Jr, Kosinski M, Dewey JE. How to score Version 2 of the SF-36 Health Survey. Lincoln, RI: QualityMetric Inc.; 2000.

31. Krystexxa prescribing information. East Brunswick, NJ: Savient Pharmaceuticals Inc. 2010. [Internet. Accessed March 22, 2012.] Available from: http://www.krystexxa.com

32. Strand V, Singh JA, Sundy J, Schumacher HR, Becker M, Edwards NL. Health-related quality-of-life (HRQOL) of patients with refractory gout and US Veterans Administration patients with gout and comorbidities (VA-Gout) is poor and comparable to that in other severe chronic conditions [abstract]. Arthritis Rheum 2008;58 Suppl:S177.

33. Bruce B, Fries JF. The Health Assessment Questionnaire (HAQ) Clin Exp Rheumatol 2005;23:S14-8

34. Fleishman JA, Cohen JW, Manning WG, Kosinski M. Using the SF-12 health status measure to improve predictions of medical expenditures. Med Care 2006;44:I54-63. 